\title{
Terrestrial gastropods (Mollusca, Gastropoda) of the Bulgarian part of the Alibotush Mts.
}

\author{
Ivailo Kanev Dedov \\ Central Laboratory of General Ecology, 2 Gagarin Str., BG-1113 Sofia, Bulgaria, e-mail: dedov@ecolab.bas.bg
}

\begin{abstract}
Dedov I.K., 2008: Terrestrial gastropods (Mollusca, Gastropoda) of the Bulgarian part of the Alibotush Mts. - Malacologica Bohemoslovaca, 7: 17-20. Online serial at <http://mollusca.sav.sk> 20-Feb-2008.
\end{abstract}

\begin{abstract}
This work presents results of two years collecting efforts within the project "The role of the alpine karst area in Bulgaria as reservoir of species diversity". It summarizes distribution data of 44 terrestrial gastropods from the Bulgarian part of Alibotush Mts. Twenty-seven species are newly recorded from the Alibotush Mts., 13 were confirmed, while 4 species, previously known from the literature, were not found. In the gastropod fauna of Alibotush Mts. predominate species from Mediterranean zoogeographic complex. A large part of them is endemic species, and this demonstrates the high conservation value of large limestone areas in respect of terrestrial gastropods.
\end{abstract}

Key words: terrestrial gastropods, distribution, Alibotush Mts., Bulgaria

\section{Introduction}

The Alibotush Mts. (other popular names: Kitka, Gotseva Planina, Slavjanka) is one of the most interesting large limestone area in Bulgaria (Fig. 1). It occupies the part of the border region between Bulgaria and Greece with maximum elevation $2212 \mathrm{~m}$ (Gotsev peak). Because of the border regime the gastropod fauna is only fragmentarily known. According to published data, 17 terrestrial gastropods are known so far. This study records 27 gastropod species for the fauna of the Mountains, so the total number of terrestrial gastropods, known from Alibotush Mts. becomes 44 (Table 1).

\section{Material and methods}

The author collected most of the materials in 2006 and 2007 from all altitude zones of the Alibotush Mountains - near villages, deciduous, mixed and coniferous forests, and high-mountain meadows and rocky terrain in sub-alpine and alpine zones of the mountain up to $2000 \mathrm{~m}$ a.s.l. (Fig. 1). Some of the samples were kindly provided by colleagues. The gastropods were collected from following localities:

Locality 1: vill. Paril, Parilski Dol (=Hambar Dere), deciduous forest, limestone rocks, on rocks, in leaflitter, seefting, hand collecting, 973-1005 m a.s.1., coll. I. Dedov, P. Subai, N. Simov.

Locality 2: road between vill. Goleshevo and vill. Paril, Fagus, 1127 m a.s.l., coll. I. Dedov.

Locality 3: vill. Goleshevo, in the village, meadow near dirt road, under stones, $1000 \mathrm{~m}$ a.s.l., coll. I. Dedov.

Locality 4: vill. Goleshevo, near Starshelitsa cave, $1000 \mathrm{~m}$ a.s.1., coll. B. Petrov.

Locality 5: vill. Goleshevo, near village, road-fork to Gotsev peak, rocky meadows, under stones, limestone, 1000-1016m a.s.1., coll. I. Dedov.
Locality 6: vill. Katuntsi, Izvorite hut, near hut, open ruderal terrain, under bark, $731 \mathrm{~m}$ a.s.1., coll. I. Dedov. Locality 7: vill. Katuntsi, tufa-gorge near village, $700 \mathrm{~m}$ a.s.1., coll. I. Dedov, N. Simov.

Locality 8: below Livade area, road between Goleshevo and Livade, limestone slope, on rocks, under stones, ecotone with deciduous/ mixed/ coniferous forest, 11001400 m a.s.1., coll. I. Dedov.

Locality 9: Livade area, nearby and western of the sheeppen, limestone slopes, on rocks, ecotone with coniferous forest, 1400-1797 m a.s.1., coll. I. Dedov.

Locality 10: southern of Livade area, road between Livade and Gotsev peak, Pinus heldreichii forest, on/under stones, limestone, 1450-1550 m a.s.l., coll. I. Dedov.

Locality 11: below/on Shabran peak, alpine meadows, limestone rocks, on rocks, under stones, 2000-2200 m a.s.1., coll. I. Dedov, B. Petrov.

Locality 12: below/on Gotsev peak, area of Suhoto ezero, on rocks, under stones, limestone, 2100-2212 m a.s.1., coll. I. Dedov, N. Simov, B. Petrov.

Locality 13: Alibotush Mts., unknown locality, coll. N. Kodzhabashev.

\section{Results and Discussion}

Among the 27 new species-records for the Mountains ( $A$. similis, P. elegans, $S$. doliolum, A. aculeata, Ch. avenacea, T. claustralis, T. cylindrica, M. obscura, Z. detrita detrita, C. laminata, B. denticulata thessalonica, P. pygmaeum, E. fulvus, D. rufa, O. cf. hydatinus, O. cf. glaber striarius, A. minor, T. budapestensis, T. kusceri, V. pellucida, O. annularis, L. cinereoniger, Lehmania sp., D. reticulatum, L. pirinensis, cf. E. strigella, H. philibinensis), 13 were confirmed (P. pusilla, Ch. tridens, Z. detrita inflata, $M$. marginata, M. pirinensis, C. schuetti, B. biplicata, $V$. bulgarica, T. serbica, A. subfuscus, X. macedonica, C. haber- 


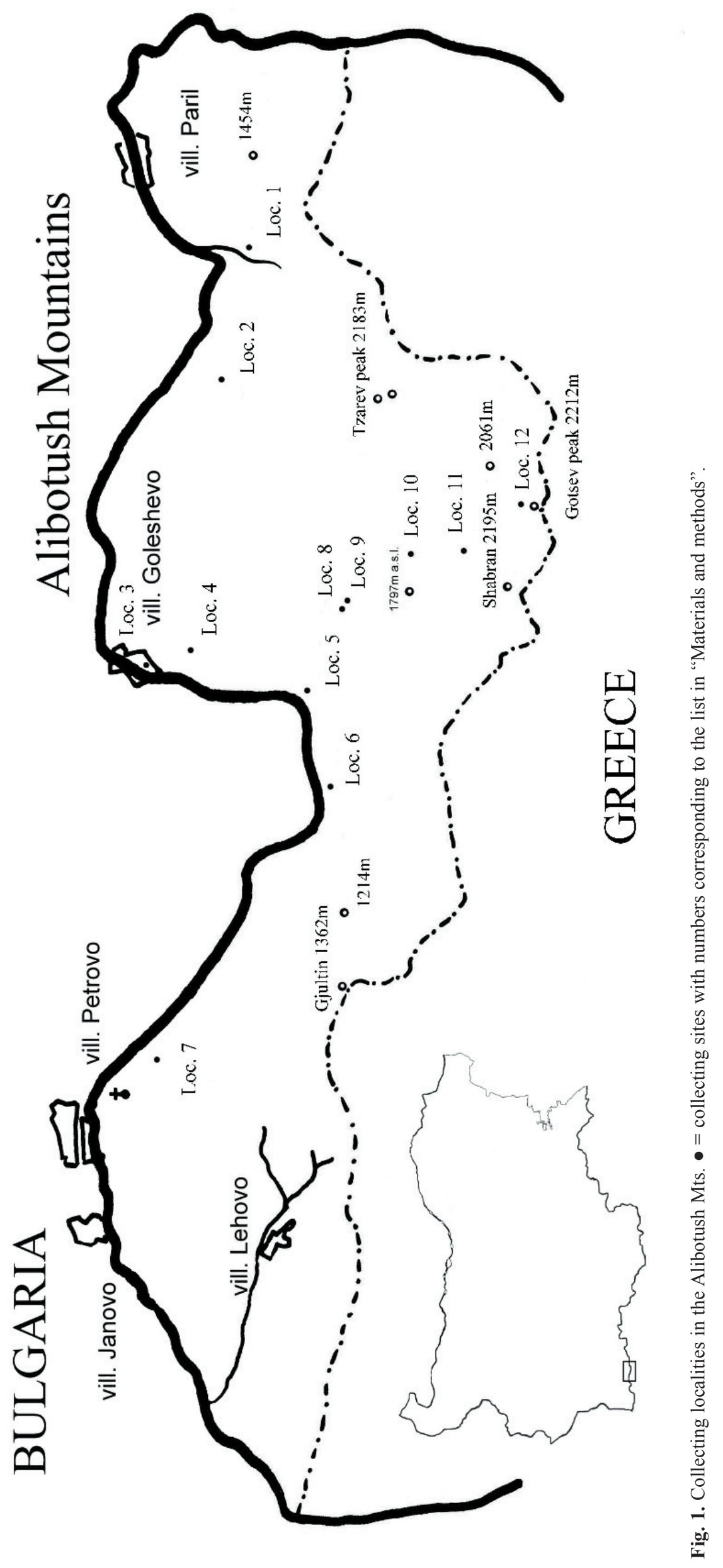


haueri, C. rumelica), while 4 species (V. alpestris, L. plicata, L. maximus, and M. cartusiana) previously known from the literature were not found during the course of the project (Table 1).

Most probably because of the relatively low altitude and warm climate, there are no high-mountain endemics typical for the alpine limestone areas of Norther Pirin Mts. (such as Wladislawia polinskii (A. J. Wagner, 1927), W. sztolcmani (A. J. Wagner, 1927), Macedonica marthae Sajó, 1968). Two species were found only in sub-alpine and alpine zones of Alibotush Mts. (O. annularis and $Z$. detrita inflata). Zebrina detrita inflata is a high-mountain subspecies, typical for alpine areas of Pirin (mainly below Vihren peak) and Alibotush Mts. This subspecies is vertically isolated from the nominal subspecies $Z$. detrita detri$t a$, which occurs in the lowest part of Alibotush. Some of the remaining gastropod species occur in the lowest areas of the mountain (28) or in all altitudinal zones (12). Three species, previously mentioned for high altitudes, in this study were found in lowest part of the mountain only ( $M$. pirinensis, C. schuetti, A. subfuscus) (Table 1) and for one ( $V$. alpestris) there is no data available.

In the gastropod fauna of Alibotush Mts., species from Mediterranean zoogeographic complex predominate (24), while Euro-Siberian species are not such numerous (19). This proportion is a result of the southern position of the Alibotush Mts. (Mediterranean biogeographic influence). The relatively high relief of the mountain supports widely distributed and cold-resisting species. The large portion of endemic species (14) demonstrates the high conservation value of large limestone areas in respect of terrestrial gastropods (Table 1).

\section{Acknowledgments}

I thank the following colleagues who have collected materials from Alibotush Mts.: Peter Subai, Nikolai Simov, Bojan Petrov, Nikolai Kodzhabashev. The research was supported by National Science Fund, Ministry of Educa- tion and Science, project No. B -1526/2005, and technically by the BioCORE project № INI-03/01.08.2005 of the Research Infrastructure Development Programme, National Science Fund, Ministry of Education and Science.

\section{References}

Damjanov S. \& Likharev I., 1975: Fauna Bulgarica IV, Gastropoda terrestria. - B.A.N., Sofia, 425 pp. (in Bulgarian).

Damjanov S. \& Pintér L., 1969: Neue Vitreini aus Bulgarien (Gastropoda: Euthyneura). - Arch. Molluskenk., 99(1-2): $35-40$.

Dedov I., 2007: On the status of the Bulgarian Pyramidula (Gastropoda, Pulmonata, Pyramidulidae). - Acta Zoologica Bulgarica, 59(2): 221-224.

HudEC V. \& VAŠÁTKo J., 1971: Beitrag zur Molluskenfauna Bulgariens. - Acta. Sc. Nat. Brno, 5(2): 1-38.

JAECKEL S.H., 1954: Zur Systematik und Faunistik der Mollusken der Nordlichen Balkanhalbinsel. - Mitt. Zool. Mus. (Berlin), 30: 54-95.

Nordsieck H., 1974: Zur Anatomie und Systematik der Clausilien, XV. Neue Clausilien der Balkan-Halbinsel (mit taxonomischer Revision einiger Gruppen der Alopiinae unb Baleinae). - Arch. Molluskenk., 104(4-6): 123-170.

PINTÉR L., 1968: Über bulgarische Mollusken. - Malak. Abhandl. Mus. Tierk.(Dresden), 2: 209-230.

URBAŃSKI J., 1964: Beiträge zur Kenntnis balkanischer Stylommatophoren. (Systematische, zoogeographische und ökologische Studien über die Mollusken der Balkan-Halbinsel. VII). - Bull. Soc. Amis. Sci. Lett. Poznan, 4: 19-56.

URBAŃSKi J., 1969: Bemerkenswerte Balkanische Stylommatophoren (Systematische, Zoogeographische und Ökologische Studien über die Mollusken der Balkan-Halbinsel. IX). - Bull. Soc. Amis. Sci. Lett. Poznań, 9 (D): 225-262.

URBAŃSKi J., 1978: Bemerkungen über balkanische Helicigonen (Gastrop. Pulm.) (Systematische, Zoogeographische und Ökologische Studien über die Mollusken der Balkan-Halbinsel XVI). - Bull. Soc. Amis. Sci. Lett. Poznan, 18: 139-149.

WikTor A., 1983: The slugs of Bulgaria (Arionidae, Limacidae, Agriolimacidae and Milacidae). - Annales zool. Warsz., 37: 71-206. 
Table 1. Species assemblage, distribution and zoogeography of the terrestrial gastropods of Alibotush Mts., SW Bulgaria.

Abbreviation: source of the data: D - Dedov (2007), DL - Damjanov \& Likharev (1975), DP - Damjanov \& Pintér (1969), HV - Hudec \& VašátKo (1971), J - Jaeckel (1954), N - Nordsieck (1974), P - Pintér (1968), PW - present work, U64 - Urbański (1964), U69 - URBAŃSKi (1969), U78 - URBAŃSKi (1978), W - WiKTOR (1983).

Abbreviation: zoogeography: BGE - bulgarian endemic, BLE - balkan endemic, PMD - ponto-mediterranean (east-mediterranean), HMD - holo-mediterranean (circum-mediterranean), LMD - lato-mediterranean (sub-meditteranean), EU - Europe, WPL - west-palaearctic, HL - holarctic.

\begin{tabular}{|c|c|c|c|c|c|}
\hline species & loc. № & source of the data & $\begin{array}{l}\text { non alpi- } \\
\text { ne areas }\end{array}$ & $\begin{array}{l}\text { sub/alpi- } \\
\text { ne areas }\end{array}$ & zoogeography \\
\hline \multicolumn{6}{|l|}{ Aciculidae (1) } \\
\hline $\begin{array}{l}\text { Acicula similis (Reinhardt, 1880) } \\
\text { Pomatiidae (1) }\end{array}$ & 1 & PW & + & - & LMD \\
\hline \multicolumn{6}{|l|}{ Orculidae (1) } \\
\hline Sphyradium doliolum (Bruguiere, 1792) & 1 & PW & + & - & PMD \\
\hline \multicolumn{6}{|l|}{ Valloniidae (1) } \\
\hline Acanthinula aculeata (Müller, 1774) & 1 & PW & + & - & WPL \\
\hline \multicolumn{6}{|l|}{ Pyramidulidae (1) } \\
\hline Pyramidula pusilla (Vallot, 1801) & $1 ; 11$ & $\mathrm{D}, \mathrm{PW}$ & + & + & EU \\
\hline \multicolumn{6}{|l|}{ Chondrinidae (1) } \\
\hline Chondrina avenacea (Bruguiere, 1792) & 1 & PW & + & - & EU \\
\hline \multicolumn{6}{|l|}{ Vertiginidae (3) } \\
\hline Truncatellina claustralis (Gredler, 1856) & 1 & PW & + & - & LMD \\
\hline Truncatellina cylindrica (Ferussac, 1807) & 1 & PW & + & - & HMD \\
\hline Vertigo alpestris (Alder, 1830) & - & DL & no data & no data & EU \\
\hline \multicolumn{6}{|l|}{ Enidae (4) } \\
\hline Merdigera obscura (Müller, 1774) & $1 ; 6 ; 8$ & PW & + & - & EU \\
\hline Zebrina detrita detrita (Müller, 1774) & $1 ; 6 ; 8 ; 9 ; 10$ & $\mathrm{PW}$ & + & - & PMD \\
\hline Zebrina detrita inflata (Kobelt, 1877) & $11 ; 12$ & J, DL, PW & - & + & BLE \\
\hline Chondrula tridens (Müller, 1774) & $1 ; 5 ; 6 ; 8 ; 11 ; 12$ & U64, PW & + & + & PMD \\
\hline \multicolumn{6}{|l|}{ Clausiliidae (7) } \\
\hline Cochlodina laminata (Montagu, 1803) & 1 & PW & + & - & EU \\
\hline Macedonica marginata (Rossmässler, 1835) & $1 ; 5 ; 8 ; 9 ; 11 ; 12 ; 13$ & J, U64, HV, PW & + & + & BLE \\
\hline Macedonica pirinensis Jaeckel, 1954 & $1 ; 11 ?$ & $\mathrm{~J}, \mathrm{~N}, \mathrm{DL}, \mathrm{PW}$ & + & $+?$ & BGE \\
\hline Carinigera schuetti Brandt, 1962 & $1 ; 8 ; 9 ; 12 ? ; 13$ & U64, DL, PW & + & $+?$ & BLE \\
\hline Balea biplicata (Montagu, 1803) & $1 ; 5 ; 6 ; 7 ; 8 ; 9 ; 10 ; 11$ & $\mathrm{U} 64, \mathrm{PW}$ & + & + & EU \\
\hline Laciniaria plicata (Draparnaud, 1801) & - & U64 & + & - & EU \\
\hline Bulgarica denticulata thessalonica (Rossmässler, 1839) & 6 & PW & + & - & BLE \\
\hline \multicolumn{6}{|l|}{ Punctidae (1) } \\
\hline Punctum pygmaeum (Draparnaud, 1801) & 1 & PW & + & - & HL \\
\hline \multicolumn{6}{|l|}{ Euconulidae (1) } \\
\hline Euconulus fulvus (Müller, 1774) & $1 ; 2 ; 8$ & PW & + & - & HL \\
\hline \multicolumn{6}{|l|}{ Daudebardiidae (1) } \\
\hline Daudebardia rufa (Draparnaud, 1805) & 1 & PW & + & - & EU \\
\hline \multicolumn{6}{|l|}{ Zonitidae (4) } \\
\hline Vitrea bulgarica Damjanov and Pintér, 1969 & $1 ; 11$ & DP, DL, PW & + & + & BLE \\
\hline Oxychilus cf. hydatinus (Rossmässler, 1838) & 4 & PW & + & - & HMD \\
\hline Oxychilus cf. glaber striarius (Westerlund, 1881) & $1 ; 8 ; 11 ; 13$ & PW & + & + & BLE \\
\hline Aegopinella minor (Stabile, 1864) & 1 & PW & + & - & EU \\
\hline \multicolumn{6}{|l|}{ Milacidae (3) } \\
\hline Tandonia budapestensis (Hazay, 1881) & 10 & PW & + & - & EU \\
\hline Tandonia kusceri (Wagner, 1931) & $3 ; 6$ & PW & + & - & BLE \\
\hline Tandonia serbica (Wagner, 1931) & 1 & W, PW & + & - & BLE \\
\hline \multicolumn{6}{|l|}{ Vitrinidae (2) } \\
\hline Vitrina pellucida (Müller, 1774) & 1 & PW & + & - & $\mathrm{HL}$ \\
\hline Oligolimax annularia (Studer, 1820) & $8 ; 11 ; 12$ & PW & - & + & PMD \\
\hline Limacidae (3) & & & & & \\
\hline Limax cinereoniger Wolf, 1803 & $8 ; 10$ & PW & + & - & EU \\
\hline Limax maximus Linnaeus, 1758 & - & DL & + & - & EU \\
\hline Lehmania sp. & 1 & PW & + & - & - \\
\hline Agriolimacidae (1) & & & & & \\
\hline Deroceras reticulatum (Müller, 1774) & 6 & PW & + & - & EU \\
\hline Arionidae (1) & & & & & \\
\hline Arion subfuscus (Draparnaud, 1805) & 10 & W, PW & + & $+?$ & EU \\
\hline Helicodontidae (1) & & & & & \\
\hline Lindholmiola pirinensis Jaeckel, 1954 & $1 ; 5 ; 6 ; 7 ; 8 ; 11$ & PW & + & + & BLE \\
\hline Hygromiidae (3) & & & & & \\
\hline cf. Euomphalia strigella (Draparnaud, 1801) & 1 & PW & + & - & EU \\
\hline Monacha cartusiana (Müller, 1774) & - & U64 & + & - & LMD \\
\hline Xerolenta macedonica Hesse, 1928 & $1 ; 8 ; 10 ; 11 ; 12$ & $\mathrm{~J}, \mathrm{P}, \mathrm{DL}, \mathrm{PW}$ & + & + & BLE \\
\hline Helicidae (3) & & & & & \\
\hline Cattania haberhaueri (Sturany, 1897) & $1 ; 8 ; 11$ & DL, PW & + & + & BLE \\
\hline Cattania rumelica (Rossmässler, 1838) & $1 ; 7 ; 8 ; 10 ; 11 ; 12$ & $\mathrm{U} 69, \mathrm{DL}, \mathrm{U} 78, \mathrm{PW}$ & + & + & BGE \\
\hline Helix philibinensis Rossmässler, 1839 & $6 ; 7 ; 8$ & PW & + & - & BLE \\
\hline
\end{tabular}

Source of the data: 27 species new for Aliboush Mts., 17 species known from literature. Non alpine areas: 41 species "+”, 1 species "no data”, 2 species “-“. Sub/alpine areas: 12 species “+”, 3 species “+?”, 1 species "no data”, 28 species "-“. Zoogeography: Medditer.: 3 - LMD, 3 - HMD, 4 - PMD, 12 - BLE, 2 - BGE; Euro-Seb.: 1 - WPL, 3 - HL, 15 - EU. 